\title{
Special feature: Econophysics 2017: synergetic fusion of econophysics and other fields of science-Part I
}

\author{
Yuichi Ikeda ${ }^{1}$ \\ Published online: 23 November 2018 \\ (C) Japan Association for Evolutionary Economics 2018
}

Mainstream economics has been recognized as one of the major academic fields today with general interest because of its impact on our daily life. What has been achieved so far has been impressive, although mainstream economics is not as successful as everyone would expect. Evidently, we need a new economic paradigm. We believe that this new paradigm is econophysics. Econophysics has been emerged as an evidence-based economics. We must recognize that for understanding a macro-economy and financial system consisting of many micro-agents, it is useless to pursue the behaviors of those micro-agents in detail. Instead, we must resort to the methods of statistical physics and network science. They provide us with the right microfoundations for macroeconomics. In addition to the application of statistical physics and network science, recently all the eyes are attracted to other fields of science which will provide synergetic fusion for econophysics to obtain the better understanding of macro-economy and financial system. For instance, the other fields of science include enterprise valuation method and alternative investment brought from the frontier of financial practices, methodologies of machine learning, and next-generation supercomputing brought from the frontier of informatics.

Workshop "Econophysics 2017: Synergetic Fusion of Econophysics and Other Fields of Science" was held on 7-8 December, 2017. The workshop addressed a number of largely unanswered questions such as how statistical physics and network science should be used to provide the right microfoundations for macroeconomics with usage of the other fields of science. More than 60 participants presented their original studies and discussed the questions and exchanged their ideas actively. The following are a brief summary of major outcomes from the workshop.

The article "The community structure of business establishments and its properties: evidence from joint patent applications", authored by Inoue (2018), provides an insight of enterprise valuation method focusing on what properties are associated with knowledge-creating communities of business establishments. By using

Yuichi Ikeda

ikeda.yuichi.2w@kyoto-u.ac.jp

1 Graduate School of Advanced Integrated Studies in Human Survivability, Kyoto University,

Sakyo-ku, Kyoto 606-8306, Japan 
Japanese patent data, the author separated Japanese business establishments into communities composed of connections through joint patent applications. It was revealed that the communities are similar in the total patents applied for, the total citations, and geographic distance and that they are dissimilar in their knowledge distance. The author claimed that the findings can benefit those who develop collaboration strategies in firms and create government policies to nurture collaborations among business establishments.

The article "Identification of conduit jurisdictions and community structures in the withholding tax network", authored by Nakamoto and Ikeda (2018), provides us an invaluable knowledge on providing the solution of international tax avoidance. Specifically, focusing on treaty shopping, which is one of the international tax avoidance schemes, authors attempt to find which jurisdictions are likely to be used for treaty shopping from the viewpoint of tax rates and reveal the relationships between jurisdictions used for treaty shopping and the others. The authors produced the withholding tax network expressed based on the withholding tax rates imposed on dividends, interest, and royalties. By computing the centralities and by detecting the communities that the jurisdictions used for treaty shopping and their community structures are identified. The authors suggested that fewer jurisdictions need to introduce more regulations for the prevention of treaty shopping worldwide.

The article "Trade network reconstruction and simulation with changes in trade policy", authored by Ikeda and Iyetomi (2018), proposed a new model to reconstruct the international trade network and associated cost network by maximizing entropy based on the local information about inward and outward trade using a machine leaning technique. The authors showed that the trade network can be successfully reconstructed using the proposed model. In addition to this reconstruction, the authors simulated structural changes in the international trade network caused by changing trade tariffs in the context of the government's trade policy. The simulations included the FOOD category and the MACHINERY category. The former simulation demonstrated that the import of FOOD from the US to Japan increases drastically by halving the import cost from the US. Meanwhile, the latter simulation showed that the exports from Japan to the US decrease drastically by doubling the export cost, while exports to the EU increased.

The article "Multiplicative random cascades with additional stochastic process in financial markets", authored by Maskawa et al. (2018), demonstrated that the multiplicative random cascade model naturally reproduced the intermittency or multifractality, which is frequently observed among the hierarchical complex systems such as turbulent fluid and financial markets. The authors investigated the validity of a multiplicative hierarchical random cascade model through an empirical study using financial data. Although the intermittency and multifractality of the time series are verified, random multiplicative factors linking successive hierarchical layers show strongly negative correlation. The authors extended the multiplicative model to incorporate an additional stochastic term, and they showed that the proposed model was consistent with all the empirical results.

The workshop achieved many of its goals, namely, highlighting the relevant results achieved by the scientific communities in the fields of econophysics, network science, machine learning, and informatics, as well as the communities of practical 
business, exploring new ideas for new methodologies, and in providing an opportunity for dialog and exchange ideas toward the new paradigm of an evidence-based economics.

The workshop was supported by the program YITP-W-17-14 of the Yukawa Institute for Theoretical Physics at Kyoto University. I am grateful to the other members of the organizing committee: Professor Hideaki Aoyama (Kyoto University), Professor Hiroshi Iyetomi (Niigata University), Professor Atsushi Ishikawa (Kanazawa Gakuin University), Associate Professor Hiroyasu Inoue (University of Hyogo), Associate Professor Takaaki Ohnishi (University of Tokyo), Associate Professor Akihiro Sato (Kyoto University), Associate Professor Wataru Sato (Nihon University), Professor Yoshi Fujiwara (University of Hyogo), Professor Jun-ichi Masukawa (Seijo University), Associate Professor Takauyki Mizuno (National Institute of Informatics), and Professor Hiroshi Yoshikawa (Risho University). We wish to express our thanks for the support of Professor Yuji Aruka (Chuo University), the staff members of Springer Japan, and the members of the conference secretariat in department of physics, Kyoto University. Finally, we would like to thank all the authors for their contributions to this special feature of Evolutionary and Institutional Economics Review.

\section{References}

Ikeda Y, Iyetomi H (2018) Trade network reconstruction and simulation with changes in trade policy. Evol Inst Econ Rev. https://doi.org/10.1007/s40844-018-0110-0

Inoue H (2018) The community structure of business establishments. Evol Inst Econ Rev. https://doi. org/10.1007/s40844-018-0116-7

Maskawa J, Kuroda K, Murai J (2018) Multiplicative random cascades with additional stochastic process in financial markets. Evol Inst Econ Rev. https://doi.org/10.1007/s40844-018-0112-y

Nakamoto T, Ikeda Y (2018) Identification of conduit jurisdictions and community structures in the withholding tax network. Evol Inst Econ Rev. https://doi.org/10.1007/s40844-018-0111-z 